\title{
DESIGN AND DEVELOPMENT OF A VISION BASED LEATHER TRIMMING MACHINE
}

\author{
M. Lanzetta, G. Tantussi \\ Department of Mechanical, Nuclear and Production Engineering, University of Pisa, Italy
}

KEYWORDS: Artificial vision, Defect detection, Path planning.

\begin{abstract}
The objective of the work described in this paper is to demonstrate a laboratory prototype for trimming the external part of a hide, assuming that the resulting machine would eventually form part of a completely automatic system in which hides are uploaded, inspected and parts for assembly are downloaded without manual intervention and prior sorting. Detailed literature and international standards are included. The expected advantages of integrating all vision based functions in a single machine, whose basic architecture is proposed in the paper, are also discussed.

The developed system is based on a monochrome camera following the leather contour. This work focuses on the image processing algorithms for defect detection on leather and the NC programming issues related to the path following optimization, which have been successfully tested with different leather types.
\end{abstract}

\section{INTRODUCTION}

Leather is animal hide or skin and it has been defined by the International Council of Tanners (Buenos Aires, 1978) and EEC directive (94/11, 23-04-1994). The most widespread commercial leather types are available from a variety of animals including cattle and calves, sheeps and lambs, goats, horses, deers and minor classes including reptiles, amphibians and aquatic animals tanned in producing countries usually for local consumption.

The trim of a hide is its shape having in mind its ultimate use (ISO 2820:1974). After preservation treatment and processing, trimming raw hides is the first step of the leather manufacturing process and is followed by nesting and cutting of components according to the size and shape of the final product, which is assembled by gluing and stitching: footwear, clothing, upholstery for automobiles and furniture, accessories (travelling and handbags, gloves, suitcases and briefcases, straps, handicraft, etc.) and technical goods (machinery belting, hydraulic packing and washers, etc.). The hide contours have a higher concentration of defects such as small holes and fringes which must be eliminated in the trimming operation.

The automation level in the leather industry is generally low, most operations are carried out manually by skilled craftsmen, so a lot can be done in this field to increase the automation level. Several attempts are available in the literature for different phases of production and most of them make extensive use of artificial vision techniques.

\subsection{STATE OF THE ART}

In [1], a vision based waterjet cutting machine is proposed, focusing on the image processing algorithms to measure the leather surface, using a fixed monochrome CCD camera with servocontrolled focal length and a backlighting system. This machine has a better resolution with 
respect to current methods based on photo-detectors. Regarding the machine evolution, examples of nesting taking into account the quality of the different hide areas and the leather homogeneity are provided. The waterjet cutting table is supposed to be separate from the inspection cell, which determines the cutting path. To fully exploit the potential of the waterjet technology several leather layers are supposed to be cut at the same time, but this increases the rejection of good material.

A similar approach has been followed by [2] which also describes a specific handling device mounted on a SCARA robot to pickup parts from a conveyor belt at variable speed up to 25 $\mathrm{m} / \mathrm{min}$ and lay them flat on a pallet with a positional accuracy of $0.5 \mathrm{~mm}$ and $1^{\circ}$. Regarding handling, the gripper design is still an open problem and it is also under study at our department [3].

In [4], a patented machine prototype is described, which is able to stitch decorative patterns on a wide range of shoe leather and synthetic shoe materials. The system uses no part-dependent specialized fittings and accepts parts of predefined shape thrown onto a conveyor in any position and orientation whose silhouette is automatically identified. Patterns are stored in "teach mode" with a mouse as key points and are interpolated by cubic splines during the stitching operation.

The recognition and orientation problems in the manipulation of shoe uppers are also dealt with in [5], which concludes emphasizing the benefit of adapting the process to the workpiece by flexible computer control, e.g. a vision based approach, as opposed to manipulating the workpiece itself to achieve the desired orientation, because floppy materials are difficult to control with sufficient reliability and accuracy during robotic handling.

Other authors [6] extract geometric features (including the square root of its area and the length of 18 radii from its centroid to its boundary at equal distance from a datum) from binarized high-resolution images for component recognition purposes ranging between 25 and $400 \mathrm{~mm}^{2}$ (most between 100 and 250) travelling at four per second.

Regarding cowhide defect detection, [7] and [8] propose a segmentation algorithm respectively using a fixed threshold and a new local contrast measure based on features extracted from the histogram of leather subimages.

Regarding the leather uniformity, international standards can be used as a reference, also for certification purposes into different classes. For instance it is required that in tests like rubbing, perspiration water and dry cleaning (ISO 11640 to 11643:1993), water spotting, migration into plasticized poly(vinylchloride), machine or mild washing (ISO 15700 to 15703:1998) or exposition to artificial light (ISO 105-B02), the color fastness be between 3 and 4 levels according to the international gray scale (ISO 105-A02), and only where the esthetic appearance is important; for instance, in the shoe industry, it is only required for uppers.

In [9], a vision system is used to correct the line drawings manually stamped on hides, by connecting the line interruptions, and to detect the quality of different areas, by recognizing the quality marks. By this, the system is able to provide a digital input to a Computer Aided Layout Design system (CALD) with a resolution of $1 \mathrm{~mm} /$ pixel in about 40 to $120 \mathrm{~s}$ per hide, depending on the drawing complexity. This operation, whose larger effort is the correction of errors coming from previous manual operations, due to the introduction of partial automation, also shows the usefulness of a system integrating all automatic functions.

The automation by a vision system of subsequent assembly operations in the shoe industry, such as skiving, which consists in reducing the thickness of the leather by the application of a 
chamfer or scarf to edges that will overlap and which would otherwise be unacceptably bulky, is described in [5]. From the above examples, it can be noticed that many aspects of the leather manufacturing process have been approached by vision, but a complete system is not yet available. A possible architecture will be proposed in the first part of this paper and the main benefits discussed.

\section{THE PROPOSED SYSTEM}

The computer integrated system described (Figure 1) can be installed at the end of a preservation processing line for trimming purposes or at the beginning of an assembly line to optimize cutting.

Hides are fed to the inspection cell where all vision based operations are carried out, followed by cells for other

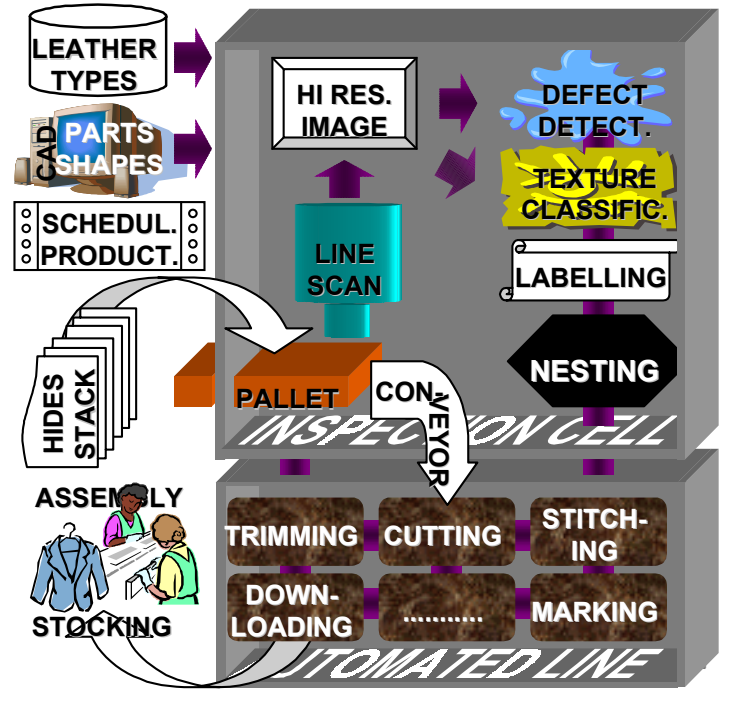

FIGURE 1. Architecture of the computer integrated automatic leather processing system. The information and material flows are respectively darker and brighter operations, such as cutting, stitching, etc., depending on the product type. Pallets on a conveyor are used to move the material through each operating cell in order to have an absolute reference with respect to all cells. All cells are connected to the inspection cell which provides the necessary data for processing.

The color texture classification is the most effective approach for defect detection, for homogeneity assessment and to label similar areas. The preferred acquisition device is a color line camera. The main benefits are:

- more repeatable lighting (on a single dimension);

- $\quad$ higher resolution with respect to matrix CCD.

The relative movement is achieved by the aforementioned conveyor. The required spatial resolution is $0.2 \mathrm{~mm} / \mathrm{pixel}$, corresponding to $41.6 \mathrm{~cm}$ with a 2048 pixel line camera [6]. For larger machines/hides, multiple cameras can be used to achieve the required resolution.

In addition to standard lighting, a structured lighting system is also used to discriminate between real defects and wrinkles due to insufficient hide stretching (especially for soft leather) after uploading. This method will also increase the system reliability for physical defect detection. A filter [7] is also used to reduce the shadows caused by the waviness.

\subsection{NESTING}

Nesting with hides has two special features: (i) the shape of the defect-free area is always different and (ii) the leather homogeneity and classification should also be taken into account.

An additional criterion is the radial distance of a part, because the leather is a fiber material. The preferential direction of fibers is radial, starting from the animal backbone, which is more homogeneous and resistant with respect to the neck, shank and belly, and which should be used for higher quality parts [10]. 
Nesting can be optimized for each hide by involving in this operation a large number of parts, e.g. all those scheduled for production during current shift. Special care will be required by the identification, handling and housing system to manage the high number of different parts to be assembled produced by the automated line. A compromise will be necessary depending on the nesting optimization level and the number of parts to be stocked. The amount of material that can be saved by optimizing a higher number of parts in the nesting operation can be easily calculated for each application of the machine and it depends on the product type.

\subsection{BENEFITS OF THE SYSTEM}

The main purpose of the system is the hide shape acquisition for the nesting optimization and the quality assessment and color measurement for leather sorting. The economic evaluation of hides and the multiple mentioned laboratory tests for color fastness (after the machine certification) would also be possible.

Additional operations required by man-made operations, like putting temporary marks on the surface of pre-cut or marking a locus for subsequent stitching (decorative in nature or part of the assembly process) would not be required anymore. Such operations were automated in [6] and [11].

The automation of leather trimming or cutting is very challenging because cycle times per component are very short (in the order of the minute) and it is important that they can be matched, but it is highly desirable for the following main benefits:

- improving the safety of the personnel working in a harmful environment and currently using sets of sharp knives at high speed;

- better material exploitation, through an optimization of the cutting path. The operator often follows shortcuts to speed up the trimming process thus increasing the rejection of good material (Figure 2);

- $\quad$ objective assessment of the various aspects of surface qualities;

- improving the process repeatability and productivity with a suitable hardware selection.

An advantage of a fixed reference, having hides in the same position on the pallet, is that parts of any shape and orientation can be extracted without additional recognition or localization algorithms for operations such as cutting, decorative stitching or handling, because these information are already stored in the system. From the literature it turns out that the resolution required for defect detection [8] [9] is 5 times higher than that required for recognition and orientation [6]. Additional benefits are:

- there is no need for training the system for recognition and orientation of each new shape processed as continuously required by the evolution of fashions;

- the elimination of recognition and orientation errors, estimated with an accuracy of 0.07 degrees and $0.2 \mathrm{~mm} / \mathrm{pixel}$ in [6]; in particular, the difference in dimensions between adjacent sizes can be small and yet the process needs to be able to discriminate between them. It should be noticed that a typical manufacturing unit might have in process say 20-40 shoe styles, each one comprising left and right, each with ten sizes and perhaps as many as twenty components in the upper alone.

\section{CURRENT WORK}

Our work is focused on the trimming operation and it is based on a specific request from the industry. A laboratory prototype of the machine with its control software has been developed. It 
is intended for small size hides, while: cattle hides can fit a rectangle up to $3 \times 4 \mathrm{~m}$. The "home" position is in the center of the working space, represented by a rectangle of $321 \times 575(\Delta \mathrm{X} \times \Delta \mathrm{Y}) \mathrm{mm}$. After the hide has been positioned, the camera is moved from the home position towards the external border in the radial direction with angle $\Theta_{0}\left(45^{\circ}\right.$ by default). To test the trimming algorithm, the camera moves around and grabs different portions of the whole hide. After acquisition each image is individually processed to de-

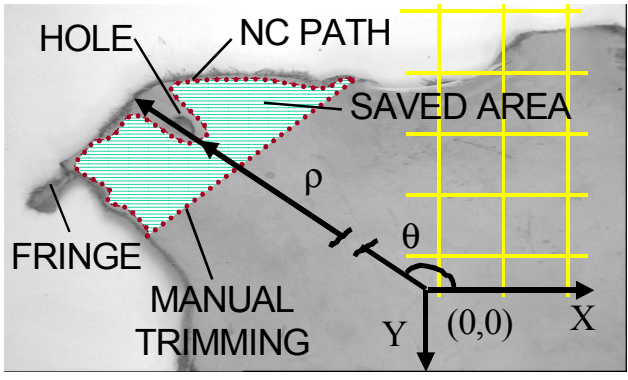

FIGURE 2. An image grabbed with leather defects, the optimal convex trimming path, the machine reference system and (part of) the control grid termine the background and the defective areas to find the trimming path. This behavior simulates the analysis of sub-images extracted from the whole hide image scanned by a line camera.

No optical distortion (estimated below the order of the pixel) compensation algorithms have been used.

\subsection{DEFECT DETECTION}

Defects on hides can be divided into three categories: ante-, post-mortem and preservation defects (ISO 2822-1:1998). They include flaws, wrinkles, scars, mites (insect bites), brand and growth marks. Hides before trimming have a ragged border with fringes and small holes. To discriminate between leather and background, the mode of a $8 \times 8$ pixel control window in the image center is compared with the mode of the background (240). To give an order of magnitude, the size of a pixel with current magnification and camera resolution is about 0.4 $\mathrm{mm} /$ pixel. With current resolution this is the minimum statistically significant size.

To determine the defective areas, the current image is divided in a regular grid of $10 \times 10$ squares (Figure 2) and the mode is computed for each. A leather area is considered defective if its gray level differs significantly from the mean of the modes of all the observed squares, after excluding those containing the background (e.g. all squares whose mode is greater then 240). The mode of a square is considered instead of the mean because it is faster to calculate and it is not affected by the presence (up to about $50 \%$ ) of pixels belonging to the background or to defective areas. In the rare event of a control window containing more than $50 \%$ of defective pixels, its weight in the calculation of the mean gray level of good pixels will be anyway low, because, in the worst case (only $1 / 4$ of the image containing leather and the rest with the background), the mean is calculated on 25 squares. From the mean of modes computed, the acceptable range of "good pixels" is calculated. It depends on the leather uniformity, in our case $(-15,+30)$.

After binarization, the leather appears as a black surface on white background and an opening (a 10 step erosion followed by a dilation) is also performed on the black area to eliminate small fringes from the contour. To determine the final contour of the good leather for trimming, the leather edge is detected by a convolution using a Laplacian mask. Considering that a binary image is input, the result is a white 1 pixel thick path on a black background. 


\subsection{PATH PLANNING}

The function to optimize in the path planning strategy is the minimization of the scrap material. The software interface is very simple. It contains one main window and several control buttons. It displays the $\mathrm{X}$ and $\mathrm{Y}$ coordinates of the camera position in real time with respect to the absolute reference system joint to the machine. At each acquisition, the points belonging to the contour are converted from pixel to $\mathrm{mm}$ and from the image reference system to the machine absolute system, considering the camera position at the time of acquisition. The Cartesian coordinates returned by the controller card are also converted in polar coordinates $\mathrm{R}$ and $\Theta$ with respect to the machine center, which can be assumed as a center of rotation in the trimming operation. Hides should be positioned in order to be centered with respect to the machine center. Considering the radial distribution of fibers and the typical hide shape, this positioning allows optimal material exploitation and avoiding undercuts in path planning.

The use of polar coordinates is very effective in this application for the following reasons.

1. The initial angular position $\Theta_{0}$ is recorded to determine the trimming end.

2. They are used to order in a sequence a set of points from a $2 \mathrm{D}$ matrix using the coordinate $\Theta$.

3. To determine the optimal convex path which excludes a hole or a fringe (in the sense of Figure 2) the most internal points between those belonging to the hide contour and those belonging to the defect edge, if any, are selected considering the coordinate R. Only the radius of points with the same $\Theta$ approximated to the second decimal digit are compared to each other.

The path polar coordinates are then converted again in Cartesian coordinates that constitute the NC input. The unit of measure is the "count" which depends on the resolution of the stepper motor and the screw axial pitch. The output of the vision system is a sequence of $2 \mathrm{D}$ coordinates that are passed on to the axes NC. The path points are 1 pixel apart from each other, so the image resolution corresponds to the path resolution. The $\mathrm{NC}$ operates in vector mode and each new point is input as a difference from the current position.

The sequence of operations described is executed in real time, while the camera follows the profile determined after previous acquisition. The path is followed having the current point in the image center. The camera field of view has been selected taking into account the required resolution and the typical defect size to allow enough visibility for sudden changes of directions. Any difference in the overlapping of subsequent images is solved by linear interpolation by the NC low level routines.

The trimming path determined by the inspection cell also allows an accurate estimation of the defect-free leather area (ISO 11646:1993) that can be used to estimate its value and to schedule the amount of raw material necessary to fulfil a certain production request from the market.

\subsection{FEATURES AND TESTING OF THE DEVELOPED SYSTEM}

The inspection cell (Figure 3) is a gantry. The leather is positioned on a bright white panel to enhance the contrast with most leather types, which are dark. Two pentaphosphor Osram lamps model Lumilux Deluxe with high frequency power supply have been positioned on the cell ceiling symmetrically along the $\mathrm{Y}$ axis. The structure is isolated from the environmental lighting. Two recirculating ball-bearing slides carry a monochrome matrix CCD camera model M50 from Jai with a Computar Telecentric lens to reduce optical distortions. 
A 4-axis numerical motion controller card from Galil model DMC-1740 controls two 2phase $95 \mathrm{~W}$ hybrid stepper motor from $\mathrm{Pa}$ cific Scientific with bipolar driver. The grabbing card is a Matrox Meteor-2MC with MIL. Both mentioned cards are installed on a PII MS Windows based PC. The vision and control program and interface have been programmed using MS Visual Basic. The geometric path computations are performed using the Mathworks Matlab 6.0 ActiveX.

The developed system has been used both to test the inspection and the trimming routines. By its motion the camera simulates the cutting head. It has been shown to be able to recognize most defects with different leather types

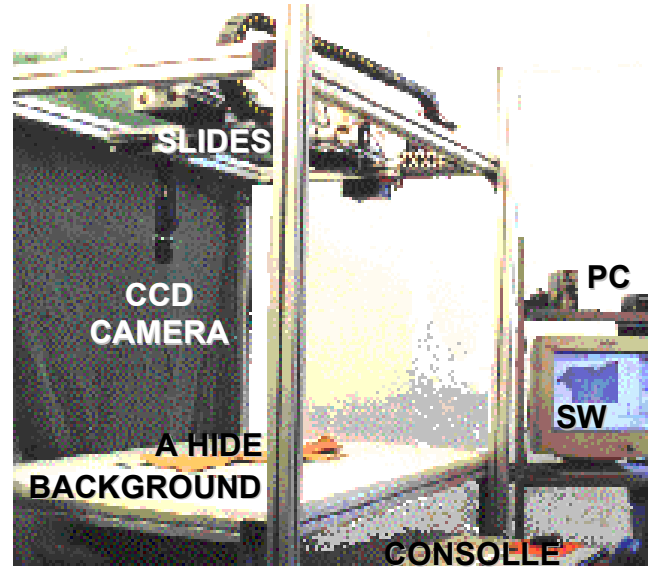

FIGURE 3. Prototype of the inspection cell and to simulate the trimming path. The surface finish and color has a strong influence on the outcome of inspection, yielding better results with brighter leather. Flaws on dark leather require higher light intensity. Highly reflective surface can create patchy saturated regions causing mis-detection.

The inspection and trimming time for a $320.000 \mathrm{~mm}^{2}$ hide with a path of $2000 \mathrm{~mm}$ is about 2 minutes (the average speed with current hardware is upper limited). The time to process each image is less than a second.

\section{FUTURE DEVELOPMENTS AND CONCLUSIONS}

The simple defect detection algorithm developed is based on the average gray level of the examined leather. In the final system an additional routine will be necessary for the classification of the leather texture in different homogenous areas. The literature on this is very rich ([7] [8] [12] [13] and the bibliography included therein). This will also allow overcoming the hypothesis of a white background that could be too stringent with the same pallet used in the cutting cell. On the other side some defects are too subtle to robustly influence the parameters of the statistical model alone, so both detection approaches cooperating will be required.

The great potential of concentrating all vision based functions in a single machine has been shown from the analysis of literature and the description of the proposed system. The technology required for its implementation is state of the art, so the next step is their integration and engineering. The main drawback of this radical approach is the low technology level of the leather industry and consequently the small target market in the short term. So further applied research is required in order to increase the cost effectiveness of a completely automatic inspection and processing system. The defect detection and the path following algorithms implemented and described in this paper can be included in such system.

\section{ACKNOWLEDGEMENTS}

The authors would like to thank Ing. Paolo Bologni for his contribution to this study. Support from Mr. Flavio Antonelli, Mr. Enzo Peroni and Mr. Salvatore Balestrino of the Department of Mechanical, Nuclear and Production Engineering, University of Pisa, are acknowledged. 


\section{REFERENCES}

1. Aranda Penaranda, J.D., Ramos Alcazar, J.A., Tomas Balibrea, L.M., Munoz Lozano, J.L., Torres Sanchez, R., (1994), Inspection and measurement of leather system based on artificial vision techniques applied to the automation and waterjet cut direct application. IEEE Int. Conf. on Systems, Man, and Cybernetics, Humans, Information and Technology, San Antonio, TX, USA, 2-5 Oct. 1994, Vol 1, 863-867, ISBN: 0-7803-2129-4.

2. Clot, R, Redarce, H.T., Betemps, M., Jutard, A., (1989), Sucker set configured by vision in real time: applicated grabbing flat and thin pieces. Proc. IASTED Int. Symp. Applied Informatics - AI '89, ACTA Press, Anaheim, CA, USA, 76-79, ISBN: 0-8898-6117-X.

3. Neri, F., (2001), Development of an automatic leather manipulation system for the tannery industry. Master Thesis (in Italian), University of Pisa, Italy.

4. Smith, D.L., Taylor, P.M., Taylor, G.E., Jolliffe, I., Reedman, D.C., (1991), Decorative stitching of randomly fed shoe parts. 91 ICAR, 5th Int. Conf. on Advanced Robotics, 'Robots in Unstructured Environments', Pisa, Italy 19-22 June 1991, Vol 1, 765-768, ISBN: 0-7803-0078-5.

5. Preece, C., Reedman, D.C., Simmons, J.E.L., Topis, S., (1994), Assembling non-rigid products in the shoe industry. IEE Computing and Control Division Colloquium on Intelligent Automation for Processing Non-rigid Products, London, UK, 19 Oct. 1994, 9/1-3, ISSN: 0963-3308.

6. Tout, N., Norton-Wayne, L., Reedman, D., (1991), Automated identification of shoe upper components. IEE Colloquium on Binary Image Processing - Techniques and Applications, London, UK, 10/1-10/5.

7. Hoang, K., Wen, W., Nachimuthu, A., Jiang, X.L., (1997), Achieving automation in leather surface inspection. Computers in Industry, Elsevier science, Vol 34, 43-54.

8. Azencott, R., Yao, J., (1994), Automated detection of cowhide defects using Markov random field techniques. Proc. 12th IAPR International Conference on Pattern Recognition, Jerusalem, Israel 9-13 Oct. 1994, Vol 1 Conf. A: Computer Vision \& Image Processing, 791-793, ISBN: 0-81866265-4.

9. Lerch, A., Chetverikov, D., (1992), Correction of line drawings for image segmentation in leather industry. Proc. 11th IAPR Int. Conf. on Pattern Recognition, The Hague, Netherlands, 30 Aug.3 Sept. 1992, Vol 1 A: Computer Vision and Applications, 45-48, ISBN: 0-8186-2910-X.

10. Manzo, G., (1999), Chimica e tecnologia del cuoio. Mediaservice, Legnano, Italy, 824 pp.

11. Preece, C., Simmons, J.E.L., Tout, N.R., Reedman, D.C., (1991), Marking and manipulation problems in the shoe industry. 91 ICAR (cited above), 786-789.

12. Lanzetta, M., Tantussi, G., Sviluppo e confronto di tecniche di controllo di qualita' di manufatti in granito (invited). Proc. "Studi e indagini sui materiali lapidei", Carrara 2000, XXI IMM, Carrara (Italy), June, 3rd 2000, 21-30.

13. Mirmehdi, M., Petrou, M., (2000), Segmentation of Color Textures. IEEE Trans. on Pattern Analysis and Machine Intelligence, Vol 22, 2, February 2000, 142-159. 